\title{
Research suggests that total hip replacement provides significant benefits, regardless of age
}

A recent study from researchers at Duke University Medical Center, North Carolina, USA, suggests that elderly patients with osteoarthritis who undergo total hip replacement are twice as likely to show improvements in physical functioning, as well as an increased ability to care for themselves, compared with peers who do not undergo the procedure.

'Osteoarthritis of the hip has a devastating impact on a patient's quality and length of life.'

Osteoarthritis is a progressive type of arthritis with close links to aging and obesity. The condition affects millions of people in the USA alone, resulting in pain, decreased mobility and an increase in the risk of falls and fractures. Hip replacements are offered to those for whom other, less invasive types of therapy such as medications and physical therapy have failed.

"Osteoarthritis of the hip has a devastating impact on a patient's quality and length of life. Our study aimed to understand how total hip replacements affect tasks people do in their everyday lives, such as bathing, dressing, walking a few blocks, shopping and preparing meals," commented one of the study's co-authors, Linda George.

The study, published in the June issue of the Journal of the American Geriatrics Society, represents the largest of its kind to date and the results suggest that there is no age limit on the benefits of hip replacements. "We found that total hip arthroplasty improves everyday life for patients and is as beneficial to people in their 80 s or 90 s as it is for someone in their 60s," states George.

Data for the study was obtained from the Medicare Current Beneficiary Survey from 1992-2003. Health data for participants who received total hip replacement $(\mathrm{n}=131)$ were compared with data for participants who suffered from osteoarthritis of the hip but did not receive total hip replacement $(\mathrm{n}=257)$. Patients were interviewed three times each year for 4 years and three measures of physical functioning were used to assess outcome: Nagi items, instrumental activities of daily living, and activities of daily living.

"We found that total hip
arthroplasty improves
everyday life for patients
and is as beneficial to people
in their 80 s or 90 s as it is for
someone in their 60 s."

The results of the study demonstrated that participants in receipt of total hip replacement demonstrated significant improvement in two of three levels of physical functioning, while the group that received no treatment experienced significant declines in physical functioning. In addition, the researchers note that the benefits are widely applicable owing to the fact that a wide range of covariates, controlled during the study, had minimal effects on receipt and outcome of total hip replacement.

Within 1 year of total hip replacement, patients who were disabled at the time of surgery had transitioned out of disability. In addition to the improved quality of life, health economists estimate the savings associated with a disability-free life to be approximately US\$50,000, including all related healthcare costs related to the disability, such as hospital stays, nursing homes and home healthcare. In contrast, reimbursement for the procedure averages US\$4000-6000.

'...fewer than $25 \%$ of patients

who could benefit from the

procedure elect to receive it.'

Total hip replacement is an invasive treatment with a long rehabilitation period; the study authors suggest that this may explain why physicians are less likely to present this option to the very old. "While the number of surgeries conducted in the [USA] has increased dramatically over the last decade, fewer than $25 \%$ of patients who could benefit from the procedure elect to receive it," notes George. However, "[physicians] should feel confident in recommending this procedure to those who are eligible for it," she argues.

"...physicians should feel confident in recommending this procedure to those who are eligible for it."

Source: George LK, Ruiz D Jr, Sloan FA: The effects of total hip arthroplasty on physical functioning in the older population. J. Am. Geriatr. Soc. 56(6), 1057-1062 (2008). 


\section{in brief}

Osteoporotic fracture risk in elderly women: estimation with quantitative heel US and clinical risk factors.

Guessous I, Cornuz J, Ruffieux C, Burckhardt P, Krieg MA: Radiology 248(1),179-184 (2008). Data from a 3-year prospective, multicenter study was used to assess the predictive value of heelbone quantitative ultrasound (QUS) in order to identify those at risk for osteoporotic fracture in 6174 Swiss women. Known risk factors for osteoporosis and fall as well as a QUS stiffness index, calculated using a QUS device, were incorporated to develop a predictive rule for osteoporotic fracture. The results demonstrated that 1464 women $(23.7 \%)$ were considered to be at lower risk for osteoporotic fracture; 4710 (76.3\%) were considered to be at higher risk. During the study period, $6.1 \%$ of the higher-risk women had an osteoporotic fracture compared with $1.8 \%$ of the women considered to be at lower risk. In addition, $90 \%$ of the women who experienced a hip fracture during the study were categorized in the higher-risk group. The researchers conclude that use of a prediction rule obtained by using QUS stiffness index alongside four clinical risk factors effectively discriminates women at higher risk of osteoporotic fracture from those at lower risk.

Can prostate-specific antigen and prostate-specific antigen velocity be used for prostate cancer screening in men older than 70 years?

Mouraviev V, Broadwater G, Sun L, Mayes JM,

Moul JW, Polascik TJ: Urology 6, 1020-1023

(2008).

The lower threshold of prostate specific antigen (PSA) and PSA velocity (PSAV) was determined in a population of men over 70 years of age.

Included in the study were 605 African-American (AA) men and 3433 non-African-American (nonAA) men. The median age for all men in the study was 75 years. The area under the curve (AUC) for PSA in AA and non-AA men was 0.84 and 0.76 , respectively; for PSAV, the AUC was 0.71 versus 0.54 for $A A$ and non-AA men, respectively. Among the AA patients, the largest relative sensitivity and specificity was achieved at the established PSA cut-point of $4.0 \mathrm{ng} / \mathrm{ml}: 85 \%$ and $71 \%$, respectively. The best cut-point in non-AA men was $3.4 \mathrm{ng} / \mathrm{ml}$, resulting in a sensitivity and specificity of 72 and $73 \%$, respectively. The researchers found that age, PSA and PSAV were significant predictors of cancer status in the AA subset; age and PSA were significant predictors in the non-AA subset. The researchers conclude that, from their data, a PSA or PSAV cutoff that would allow safe discontinuation of prostate cancer screening was not apparent.

\section{UK study suggests rates of selected sexually transmitted infections are increasing in the over-45s}

In a recent study that examined the distribution of selected sexually transmitted infections (STIs), (chlamydia, genital herpes, genital warts, gonorrhea and syphilis), data was obtained from people attending Genitourinary Medicine (GUM) clinics in the West Midlands, UK.

During the study period between 1996 and 2003, 4445 STI episodes were recorded among older persons (those aged 45 years or older). The rate of STIs in older persons was found to have more than doubled in 2003 compared with 1996 from 16.7 per 100,000 population to 36.3 per 100,0000 , representing a significantly higher increase in STIs in the older compared with younger patients. In addition, the rate for all of the selected STIs in the study had significantly increased by 2003 , with genital warts accounting for almost half of these diagnoses. Herpes was found to be the second most common, accounting for approximately a fifth of diagnoses.

The results demonstrated that, overall, people aged between 55 and 59 years of age were significantly more likely to be affected by an STI, with men more highly represented than women. Babtunde Olowokure, one of the study's co-authors from the HPA's regional surveillance unit in Birmingham, UK, remarked, "Sexual health strategies have rightly focused on the under-25s, but our results indicate that sexual risk-taking behaviour is not confined to young persons but is also an increasing trend in the over-45s."

Commenting on the possible reasons for the high rate of STIs observed in older people,
Olowokure noted, "Older people are increasingly likely to be single or undergoing relationship changes and are less likely to consistently use condoms, perhaps because the risk of pregnancy no longer exists. Increased international travel, internet dating, new drugs to counter erectile dysfunction and overlapping sexual networks may also be factors."

Julie Bentley, the chief executive of the sexual health charity FPA, remarked: "We've also noticed a rise in the numbers of over- $45 \mathrm{~s}$ phoning our helpline. Tragically, the sexual health of men and women of this age group is largely neglected and its something we are increasingly concerned about. Bentley went on to add, "Services are geared towards young people, campaigns are targeted at the under 25 's so over$45 \mathrm{~s}$ think that sexual health has nothing to do with them and don't even know when they're taking risks."

Writing in the journal of Sexaually Transmitted Infections, the researchers conclude that their results provide evidence to support the development and implementation of "evidencebased multifaceted sexual health programmes that, while aiming to reduce STI transmission among all age groups, should include interventions aimed specifically at older persons and address societal and healthcare attitudes, myths and assumptions regarding sexual activity among older people."

Source: Bodley-Tickell AT, Olowokure B, Bhaduri $S$ et al:: Trends in sexually transmitted infections (other than HIV) in older persons: analysis of data from an enhanced surveillance system. Sex. Transm. Infect. (2008) (Epub ahead of print). 


\section{Women over 90 may be more at risk of dementia than their male peers}

The prevalence of dementia has been demonstrated to increase between the ages of 65 and 85 . However, there has been little research to demonstrate whether or not this trend increases past the age of 90 years. A recent study, published in the journal Neurology, looked at 911 participants from the $90+$ study, a population-based study of aging and dementia in people aged 90 and above.

In-person examinations as well as telephone and informant questionnaires were conducted in order to diagnose dementia. The results demonstrated that the overall prevalence of all-cause dementia was higher in women compared with men at 45 and $28 \%$, respectively. In women, the prevalence of dementia approximately doubled every 5 years after 90 years of age. However, the same trend was not observed in men. In addition, while a lower prevalence of dementia was associated with higher education in women, this was not true for men.

"As more and more people reach age 90 , our findings provide further evidence that more needs to be done to provide adequate resources to care for the increasing number of very old people with memory problems."

The reasons for the higher prevalence of dementia observed in women remain to be elucidated. However, elderly women are more prone to heart disease and stroke compared with men, both of which are risk factors for stroke.

A recent report by the King's Fund suggested that, in the UK, the prevalence of dementia was likely to rise sharply as a result of the aging population. The report predicted that the total bill for care would more than double to over $£ 35$ billion a year, as the number of people with the illness rises past 900,000. The aging population means that studies investigating the very old hold a great significance. Maria Corrada, the study's lead author, commented, "As more and more people reach age 90 , our findings provide further evidence that more needs to be done to provide adequate resources to care for the increasing number of very old people with memory problems."

Commenting on the research, The Alzheimer's Society noted there was now an urgent need to find out more about how gender affected the likelihood of dementia.

Source: Corrada MM, Brookmeyer R, Berlau D, Paganini-Hill A, Kawas $\mathrm{CH}$ : Prevalence of dementia after age 90. Results from The 90+ Study. Neurology (2008) (Epub ahead of print).

\section{Ability of quantitative ultrasound to predict fracture risk in the elderly may be modified by previous fracture status}

Quantitative ultrasound (QUS) has been shown to predict the risk of fracture in various populations. However, a recent study suggests that in very frail older people, this ability may be modified by the presence of previous fracture(s).

Writing in the journal Osteoporosis Inernational, Jason Chen and colleagues note that, "a history of fracture has been reported to predict future fractures and it is thought that this risk factor influences the risk of fracture through the same pathway (skeletal fragility) that QUS measures.”

The researchers assessed bone strength using QUS and clinical risk factors at baseline for 1982 institutionalized older people with a mean age of 85.7 years. Follow-up for fractures was carried out at 2 years from baseline, using $x$-ray reports. A total of $70 \%$ of participants enrolled in the study used a walking aid and $45 \%$ reported a history of fracture.

Mean follow-up time in the study was 1.64 years, and during this time, 335 participants suffered one or more fractures. However, the rate of fracture was significantly higher in those who had experienced a previous fracture compared with those who had no history of previous fracture at 16.0 versus 9.2 fractures per 100 person-years, respectively.

In addition, the researchers also found significant associations between fracture risk and QUS parameters (broadband ultrasound attenuation and velocity of sound) in those with no prior fractures; however, this was not seen in those with a fracture history.

The researchers conclude that in very frail older people, QUS measurements may be more useful for assessing fracture risk in those with no history of fracture after 50 years of age, recommending that "if QUS is used to identify individuals who should benefit from preventive interventions, it may be better focused on elderly participants who have not had a previous fracture."

Source: Chen JS, March LM, Cumming RG et al: : Role of quantitative ultrasound to predict fracture among institutionalised older people with a history of fracture. Osteoporos. Int. (2008) (Epub ahead of print). 